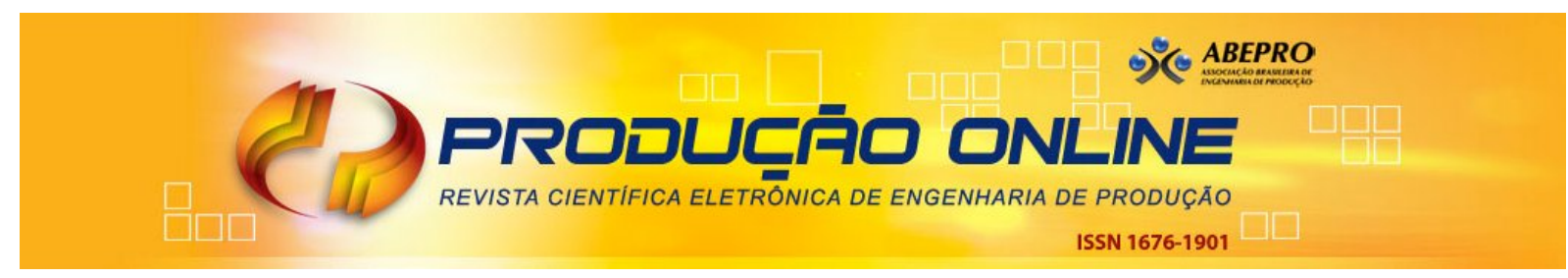

\title{
ADOÇÃO DO ECODESIGN EM EMPRESAS INOVADORAS NO BRASIL: LEVANTAMENTO E ANÁLISE DOS PRINCIPAIS ESTÍMULOS
}

\section{ADOPTION OF ECODESIGN IN INNOVATIVE COMPANIES IN BRAZIL: SURVEY AND ANALYSIS OF THE MAIN STIMULUS}

\author{
João Victor Rojaz Luiz* E-mail: joao.rojas@feb.unesp.br; \\ João Leonardo de Andrade* E-mail: prod.joaoleonardo@gmail.com; \\ Daniel Jugend* E-mail: daniel@feb.unesp.br \\ Sergio Luis da Silva** E-mail: sergiol@ufscar.br \\ Octaviano Rojas Luiz* E-mail: orojasluiz@yahoo.com.br \\ Fernando Bernardi de Souza* E-mail: fbernardi@feb.unesp.br \\ *Universidade Estadual Paulista Júlio de Mesquita Filho (UNESP), Bauru, SP \\ **Universidade Federal de São Carlos (UFSCAR), São Carlos, SP
}

Resumo: Embora o interesse pela questão ambiental tenha ganhado importância na área de gestão de operações nos últimos anos, observa-se ainda uma lacuna em pesquisas nacionais. Visando contribuir com esta área, a presente pesquisa tem como objetivo levantar e compreender quais são os principais estímulos para a adoção do ecodesign em empresas no país. Foi realizada uma pesquisa de levantamento do tipo survey em 79 firmas pertencentes a setores inovadores e envolvidas em atividades de desenvolvimento de novos produtos. Dentre os principais resultados observou-se que 0 atendimento aos requisitos legais e possibilidades de novas oportunidades de negócios se sobressaem como os principais estímulos para a adoção do ecodesign. A utilização do ecodesign como elemento para a redução de custos também é incentivo considerado relevante pelas empresas. Por outro lado, o "benefício ao meio ambiente" é tido como o estímulo menos atrativo.

Palavras-chave: Sustentabilidade ambiental. Processo de desenvolvimento de produtos. Ecodesign. Estímulos para a adoção. Pesquisa tipo survey.

\begin{abstract}
Although concern about the environmental issues has gained prominence in the field of operations management in recent years, a research gap still exists in companies operating in Brazil. In order to contribute to this area, this research aims to explain and understand the main stimulus for the adoption of ecodesign in Brazil. To this end, a survey was carried out in 79 firms belonging to innovative sectors and involved in new product development activities. Among the main results, it was observed that compliance with legal requirements and new business opportunities stood out as the main stimulus for the adoption of ecodesign. The possibility of using ecodesign as an element of cost reduction was also a stimulus considered relevant by companies. On the other hand, the stimulus "benefit to the environment" was considered the least attractive.
\end{abstract}

Keywords: Environmental sustainability. Product development process. Ecodesign. Incentives for adoption; Survey research.

\section{INTRODUÇÃO}

Diversas pesquisas recentes têm chamado a atenção para a necessidade das 
organizações integrarem a sustentabilidade ambiental em seus esforços relacionados à gestão do processo de desenvolvimento de produtos (BRONES et al., 2014; EPPINGER, 2011; KUBOTA et al., 2014). O interesse pela questão ambiental vem crescendo, principalmente, devido as pressões de governos e consumidores (DALHAMMAR,2016) e, diante da escassez dos recursos naturais e aumento dos índices de poluição (WALKER et al., 2008). Desta forma, no âmbito do processo de desenvolvimento de produtos muitas empresas também estão incorporando características ambientalmente "amigáveis" em seus projetos de produtos (BORDCHARDT et al., 2010; VERCALSTEREN, 2001).

Nos últimos anos muitos trabalhos em gestão ambiental, desenvolvimento de novos produtos e em gestão de projetos (BRONES et al., 2014; LUIZ et al., 2016) têm investigado e analisados práticas para o desenvolvimento de produtos ambientalmente sustentáveis. Dentre essas práticas destaca-se o ecodesign (VARANDAS JUNIOR, 2014; DEKONINCK et al., 2016). De maneira geral, o ecodesign pode ser definido como uma abordagem na qual os objetivos ambientais são avaliados em conjunto e de maneira integrada aos objetivos do projeto do produto (FIKSEL, 1996). Mais especificamente, Dangelico (2005) pondera que os produtos ambientalmente sustentáveis são aqueles projetados com a preocupação de diminuir o impacto ambiental ao longo de todo o seu ciclo de vida; desde a extração e aquisição da matéria prima, através da produção de energia e materiais, manufatura, uso até a disposição final, descarte ou retorno do produto à empresa produtora.

É reconhecida a importância da aplicação de princípios e de métodos do ecodesign como meio para se integrar a preocupação ambiental desde a etapa de projeto, visando à otimização no ciclo de vida do produto (BRONES; CARVALHO, 2015; BYGGETH; HOCHSCHORNER, 2006; LUIZ et al., 2016). Segundo Van Hemel e Cramer (2002), a opção pela aplicação do ecodesign é derivada de estímulos (externos e internos) e barreiras que as organizações se defrontam, isto é, para a adoção do ecodesign pelas firmas é importante que haja maior quantidade de estímulos do que barreiras. Sendo que estes estímulos para a adoção do ecodesign podem ser externos e internos, e não se limitam apenas a benefícios ambientais, mas também àqueles de caráter social, econômico e tecnológico (VAN HEMEL; 
CRAMER, 2002).

Apesar da reconhecida importância que circunda o tema estímulos à adoção do ecodesign, cujo conteúdo é tratatado principalmente pela literatura internacional (VAN HEMEL; CRAMER, 2002; COLLADO-RUIZ; OSTAD-AHMAD-GHORABI, 2013), são poucos os estudos quantitativos e nacionais que buscam investigar este tema em uma ampla gama de firmas que atuam no país. Visando contribuir com essa área de pesquisa, este artigo se concentra em apresentar e analisar os principais estímulos para a adoção do ecodesign em 79 empresas que desenvolvem produtos e que são consideradas inovadoras no país. Para avaliar e analisar a adoção de ecodesign, bem como os seus principais estímulos, foi realizada pesquisa quantitativa de levantamento do tipo survey em empresas que no Brasil pertencem a setores considerados dinâmicos em termos tecnológicos e que, ao mesmo tempo, possuam atividades de desenvolvimento de novos produtos.

Para isso, após esta introdução o artigo inicialmente apresenta breve revisão teórica. Posteriormente, são explicados os procedimentos de método empregados. $\mathrm{O}$ item quatro apresenta e discute os resultados obtidos. Finalmente, as principais contribuições, limitações do trabalho e propostas de pesquisas futuras são expostas no item de conclusões do artigo.

\section{ECODESIGN E ESTÍMULOS À ADOÇÃO DE PRÁTICAS AMBIENTAIS}

A sustentabilidade ambiental é reconhecidamente tema relevante para diversas áreas gerenciais, tais como inovação, operações e desenvolvimento de novos produtos (PORTER; VAN DER LINDE,1995; PUJARI, 2006; SELLITO et al., 2013). Além de gerar vantagens aos stakeholders, alguns estudos indicam que a dimensão ambiental adequadamente integrada ao processo de desenvolvimento de produtos pode trazer benefícios, como aumento da eficiência dos recursos, melhoria da imagem corporativa, aumento das vendas e de market share e maior capacitação em novas tecnologias (DANGELICO et al., 2013), bem como melhorar o desempenho de mercado (GONZÁLEZ-BENITO; GONZÁLEZ-BENITO, 2006; PUJARI, 2006) e operacional (JABBOUR et al., 2015).

Uma vez que esta diretamente relacionada com a inserção de preocupações 
ambientais em atividades de projeto do produto (JABBOUR; JABBOUR, 2013; PIGOSSO et al., 2013), a adoção do ecodesign é elemento fundamental para empresas que desenvolvem produtos e que busquem melhores níveis de desempenho em sustentabilidade ambiental (MAGNAGO et al., 2012; DEKONINCK et al., 2016). Embora o termo ecodesign seja amplamente difundido e fundamentado pela ISO 14006-2011 (STANDARD, ISO 14006:2011), termos similares ainda são usados, como design for environment, green product development, eco-innovation ou environmentally conscious design (BAUMANN et al., 2002; FIKSEL, 1996; LUIZ et al., 2016). A multiplicidade de termos usados para a definição do conceito e sua expansão impõe certas dificuldades para as pesquisas em base de dados e, também, para a análise quantitativa da literatura no tema. Entretanto, dentro de uma interpretação abrangente, diversos termos têm significados equivalentes (BRONES; CARVALHO, 2015), e no presente artigo ecodesign será empregado representando todos os demais.

Pode se definir ecodesign como o esforço sistemático para melhorar o perfil ambiental do produto em todas suas fases do ciclo de vida, incluindo a extração da matéria prima, uso do produto, reciclagem e disposição final (VAN HEMEL; CRAMER, 2002). Outra definição utilizada é aquela fornecida pelo padrão ISO 14006 (INTERNATIONAL STANDARD, 2011), em que o ecodesign é a integração de aspectos ambientais ao desenvolvimento e projeto do produto. Para o bom desempenho desta integração, os fatores ambientais devem ser especificados já nas fases iniciais do processo de desenvolvimento de produtos, e estejam alinhados de acordo com as opções e posicionamento estratégicos da empresa (JOHANSSON, 2002), isto é, na macrofase de pré-desenvolvimento (ROZENFELD et al., 2006). Isso porque, segundo Graedel e Allenby (1995), 60\% a 80\% dos impactos ambientais causados em todo ciclo de vida do produto são estabelecidos nas fases iniciais do seu desenvolvimento, assim as melhores oportunidades de melhorias tendem a ocorrerem nesta etapa.

De acordo com González-Benito e González-Benito (2006), em termos gerenciais, o ecodesign refere-se a uma prática operacional de gestão ambiental focada no desenvolvimento de novos produtos. Esta prática envolve esforços de considerar, já na etapa de projeto, elementos como a substituição de materiais e 
componentes poluentes; a redução do consumo de recursos e de geração de resíduos durante a produção, uso e a distribuição do produto; e também aspectos como desmontagem, reutilização e reciclagem (JABBOUR et al., 2015). Stevels (1999) ressalta ainda que atividades que focam a redução dos efeitos ambientais, como o ecodesign, além de ocasionar redução de custos, tendem a promover também outras melhorias sensíveis para os consumidores destes produtos, como por exemplo: redução do peso do produto, redução do volume das embalagens, redução de materiais e a redução de energia, por exemplo.

Os princípios que direcionam o ecodesign podem ser agrupados em estratégias que levam em conta todas as etapas do ciclo de vida do produto (VAN HEMEL; CRAMER, 2002), e envolvem aspectos como: a seleção de materiais de baixo impacto ambiental, a redução do uso de materiais, otimização de técnicas de produção, a otimização do sistema de distribuição, a redução do impacto ambiental durante o uso, otimização do tempo de vida inicial do produto, otimização do tempo de vida ao final do ciclo e o desenvolvimento de novos conceitos de produto. Nesta mesma linha e baseado em revisão teórica, Kubota et al. (2014) agrupou diversas práticas em dez princípios do ecodesign, são eles: escolha de materiais com baixo impacto ambiental, buscar a simplicidade ou modularidade do projeto do produto, incinerar resíduos ao invés de aterrá-los, reduzir a demanda de energia, usar fontes de energia renováveis, desenvolver produtos multifuncionais, desenvolver produtos com maior durabilidade, recuperar a embalagem do produto, evitar o uso de substâncias perigosas e, finalmente, empregar a abordagem de prevenção de acidentes.

Borchardt et al. (2010), em estudo realizado em uma empresa de confecção de calçados, observaram que os principais resultados obtidos por meio da aplicação do ecodesign no reprojeto de um item de calçado foram a redução no custo de $10 \%$ no produto final, diminuição dos resíduos gerados, redução na utilização de material fóssil e a melhoria na imagem da empresa perante o mercado.

Também são diversos os estudos que recomendam a aplicação de métodos específicos para auxiliar as empresas para a adoção do ecodesign (BOVEA; PÉREZ-BELIS, 2012; BRONES; CARVALHO, 2015). Dentre os métodos associados, são amplamente mencionados pela literatura a análise do ciclo de vida de produtos, 
environomental - quality function deployment (EQFD), a matriz energia, material e toxidade; environmental failure mode effects analysis (E-FMEA), e o ecodesign checklist (BOVEA; PÉREZ-BELIZ, 2012; BYGGETH; HOCHSCHORNER, 2006; KNIGHT; JENKINS, 2009; LU et al., 2011).

No entanto, existem barreiras reconhecidas e que devem ser transpostas para a adoção do ecodesign (VAN HEMEL; CRAMER, 2002; AMMENBERG; SUNDIN, 2005; POULIKIDOU et al., 2014). Muitas delas se relacionam ao fato de que muitos de seus princípios não são vistos como responsabilidade do setor ou funcionário (conflito de requerimento funcional), não é evidenciado um benefício ambiental claro para a empresa ao se implantar o ecodesign, ou ainda não se consegue encontrar soluções alternativas disponíveis no desenvolvimento do produto e que sejam melhores ambientalmente (VAN HEMEL; CRAMER, 2002). Outros obstáculos relevantes encontrados na literatura são: problemas de comunicação entre funcionários e entre empresas, complicações técnicas, culturas organizacionais desfavoráveis a adoção de práticas de sustentabilidade, falta de conhecimento e recursos insuficientes (AMMENBERG; SUNDIN, 2005). Os próprios métodos e ferramentas de ecodesign podem representar barreiras, pois podem apresentar dificuldade de coleta de dados e de operacionalização, em alguns casos sendo muito simplificadas, em outros muito complexos. Estas barreiras podem ser atenuadas quando existe um processo de desenvolvimento de produtos estruturado e conhecimentos compartilhados sobre gestão ambiental e ecodesign (POULIKIDOU et al., 2014).

Em contraposição, podem-se apresentar possíveis motivadores ou aspectos de estímulos que impulsionam as empresas a adotarem o ecodesign em seus esforços de desenvolvimento de novos produtos. Dentre estes direcionadores, são reconhecidos os estímulos externos e internos às organizações (VAN HEMEL; CRAMER, 2002; DANGELICO, 2015). Demandas oriundas do mercado, regulamentações governamentais e iniciativas de setores industriais específicos são os estímulos externos tidos como mais influentes. Já os estímulos internos reconhecidos pela literatura como relevantes são o potencial de aumento de oportunidades de inovação, na qualidade do produto e das oportunidades de atuação em novos mercados (VAN HEMEL; CRAMER, 2002). Esses e outros 
estímulos com destaque na literatura são apresentados sinteticamente no Quadro 1.

Quadro 1 - Estratégias de ecodesign e a presença dos principais estímulos externos e internos.

\begin{tabular}{|c|c|c|c|c|c|c|c|c|c|}
\hline 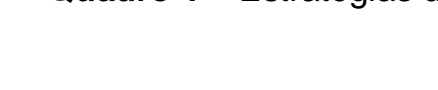 & & $\begin{array}{l}\text { Estímu } \\
\text { extern }\end{array}$ & & & & mulos & ernos & & \\
\hline Estratégias & 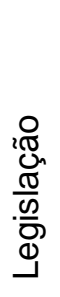 & 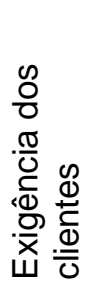 & 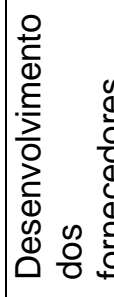 & 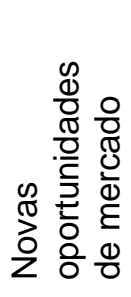 & 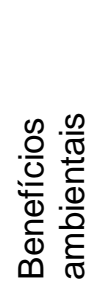 & 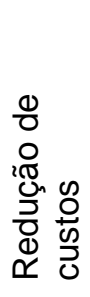 & 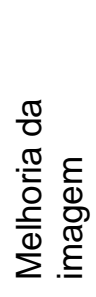 & 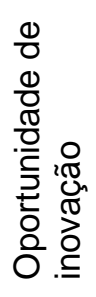 & 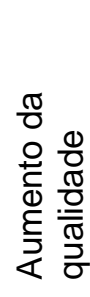 \\
\hline $\begin{array}{l}\text { Seleção de materiais de } \\
\text { baixo impacto }\end{array}$ & $X$ & $X$ & $X$ & $X$ & $X$ & $X$ & $X$ & & \\
\hline $\begin{array}{c}\text { Redução do uso de } \\
\text { materiais }\end{array}$ & $X$ & 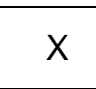 & & & $X$ & $X$ & & & \\
\hline $\begin{array}{l}\text { Otimização de técnicas de } \\
\text { produção }\end{array}$ & $X$ & & & & $X$ & $X$ & & & \\
\hline $\begin{array}{c}\text { Otimização do sistema de } \\
\text { distribuição }\end{array}$ & $X$ & $X$ & $X$ & & $X$ & $X$ & & & \\
\hline $\begin{array}{c}\text { Redução do impacto } \\
\text { ambiental durante o uso }\end{array}$ & $X$ & $X$ & $X$ & $X$ & $X$ & $X$ & $X$ & & \\
\hline $\begin{array}{l}\text { Otimização do tempo de } \\
\text { vida inicial do produto }\end{array}$ & $X$ & $X$ & & $X$ & $X$ & $x$ & $x$ & & $X$ \\
\hline $\begin{array}{l}\text { Otimização do tempo de } \\
\text { vida do produto ao final do } \\
\text { ciclo }\end{array}$ & $X$ & $X$ & & $X$ & $X$ & $X$ & $X$ & & \\
\hline $\begin{array}{l}\text { Desenvolvimento de novos } \\
\text { conceitos de produto }\end{array}$ & & & & $X$ & $X$ & & $X$ & $\mathrm{X}$ & \\
\hline
\end{tabular}

Com base no quadro 1, a análise da presença dos estímulos para a adoção dos princípios de ecodesign pode servir também de suporte ao governo, intermediários e empresas com o objetivo de ajudar a discernir quais estímulos se relacionam a cada estratégia do ecodesign (VAN HEMEL; CRAMER, 2002). Além disso, Vercalsteren (2001) recomenda que anterior à adoção do ecodesign em uma organização, é importante que se realize uma análise da viabilidade de sua aplicação, a qual se deve avaliar fatores internos como a motivação de funcionários e o impacto das mudanças que a adoção do ecodesign irá gerar nos processos de negócios, especialmente no de desenvolvimento de novos produtos. Além da avaliação de fatores externos como o comprometimento dos fornecedores e a 
demanda do mercado por produtos verdes (Vercalsteren, 2001). A avaliação desses elementos pode indicar se a empresa esta efetivamente estimulada e preparada para adotar o ecodesign.

Após esta breve revisão teórica, a seção seguinte apresenta os procedimentos de pesquisa adotados neste trabalho.

\section{MÉTODO DE PESQUISA}

Para identificar e analisar os principais estímulos para a adoção de ecodesign em empresas inovadoras e que atuam no país, foi realizada pesquisa quantitativa de levantamento do tipo survey. A pesquisa do tipo levantamento é recomendada e usada com frequência na área de gestão de operações (FORZA, 2002). Nesta abordagem, o pesquisador avalia uma amostra da população acerca de um problema a ser investigado, extraindo conclusões das informações sobre os indivíduos ou sobre os ambientes dos quais fazem parte ( MIGUEL; HO, 2010). Segundo a classificação de Forza (2002), o levantamento realizado neste estudo se enquadra como uma survey descritivo, pois tem como objetivo entender a relevância de certo fenômeno, sem propriamente desenvolver ou testar uma teoria, mas fornecendo resultados para a construção ou refinamento de teorias já existentes. Sendo que justamente os resutados desta pesquisa visam contribuir com um maior entendimento sobre os fatores que estimulam as empresas a adotarem o ecodesign.

O banco de dados usado para levantamento de dados reuniu empresas de setores considerados inovadores pelo último relatório da PINTEC (Pesquisa de Inovação Tecnológica), tais como: eletrônico, químico, aviação, óptico, aeronáutico e automação industrial (IBGE, 2013). Para a finalidade desta pesquisa, consultaramse empresas destes setores cadastradas na Associação Brasileira da Indústria Elétrica e Eletrônica, e na Câmara Americana de Comércio Brasil - Estados Unidos. Visando a operacionalização deste levantamento, foi elaborado um questionário estruturado (questões fechadas) não disfarçado, cujas respostas utilizaram, majoritariamente, a escala Likert de sete pontos (1 a 7, de discordo totalmente a concordo totalmente) para avaliar a intensidade de concordância ou discordância do entrevistado com respeito a cada variável. Os dados gerados com a pesquisa foram 
em sua maioria dados qualitativos ordinais, visto que as variáveis estudadas envolveram o grau de concordância com as afirmações presentes no questionário.

O instrumento de pesquisa (questionário) foi elaborado a partir de revisão da literatura a respeito de ecodesign e desenvolvimento de produtos ambientalmente sustentáveis. As variáveis utilizadas para se identificar os estímulos para a adoção do ecodesign foram baseadas, principalmente, no trabalho de Van Hemel e Cramer (2002). Escolheu-se este trabalho (VAN HEMEL e CRAMER, 2002) como orientador para a confecção das questões de pesquisa empregadas, pois se trata de um dos artigos mais citados mundialmente em bases de dados com o Scopus e Web of Science e que investiga as temáticas ecodesign e estímulos a adoção do ecodesign.

Como forma de melhorar a qualidade e atestar a validade das questões presentes no instrumento de pesquisa, realizou-se um pré-teste com três professores das áreas de desenvolvimento de produtos e gestão ambiental, e dois profissionais com experiência em gestão de projetos e atuantes em empresas com atividades sistemáticas de desenvolvimento de produtos. Com relação ao feedback do pré-teste, foram levantadas e incorporadas sugestões de melhoria no instrumento final de pesquisa e não houve dúvidas com relação ao conteúdo do questionário. O gerenciamento do envio do questionário às empresas participantes foi feito por meio de um site. Este site hospedou o instrumento de coleta de dados e nele foi possível cadastrar a população de empresas e enviar o link para o questionário. Ao receber o link, o respondente era direcionado para o questionário no ambiente virtual de pesquisa.

Apenas foram considerados válidos os questionários de empresas que assinalaram que efetivamente estão envolvidas com atividades de desenvolvimento de produtos. Obteve-se, por fim, uma amostra válida de 79 empresas. A maioria dos respondentes desta amostra (aproximadamente 80\%) ocupa os seguintes cargos nas empresas: presidente/proprietário, diretor, engenheiro, supervisor e coordenador, ou seja, cargos diretamente envolvidos com a tomada de decisão, gestão ou execução de etapas do processo de desenvolvimento de produtos. Os dados coletados foram submetidos à análise estatística descritiva, obtendo-se os valores de média, mediana e desvio-padrão para cada uma das variáveis relativas às assertivas do questionário. 
A Tabela 1 apresenta a classificação das empresas respondentes por setor de atuação.

Tabela 1 - Frequência de empresas por setor de atuação

\begin{tabular}{lcc}
\hline \multicolumn{1}{c}{ Setor } & $\begin{array}{c}\text { Frequência } \\
\text { absoluta }\end{array}$ & $\begin{array}{c}\text { Frequência relativa } \\
(\%)\end{array}$ \\
\hline Indústria Eletrônica & 15 & 19,0 \\
Indústria Metal Mecânica & 12 & 15,2 \\
Informática, Software ou & 12 & 15,2 \\
Hardware & 9 & 11,4 \\
Indústria Química e Petroquímica & 7 & 8,9 \\
Automação Industrial & 6 & 7,6 \\
Indústria Automotiva & 4 & 5,1 \\
Médico-Hospitalar & 3 & 3,8 \\
Geração Transmissão e & 3 & 3,8 \\
Distribuição de Energia & 8 & 10,0 \\
Telecomunicões & & \\
Outros: & & \\
\hline
\end{tabular}

Os setores apontados na alternativa "outros" foram de empresas pertencentes aos seguintes setores: aeronáutico, máquinas agrícolas, automação predial, segurança patrimonial e cabeamento estruturado, instalações elétricas industriais, eficiência energética e de energia solar, ou seja, tratam-se de firmas pertencentes a setores considerados inovadores.

A Tabela 2 apresenta a frequência de empresas de cada porte. Para isso adotou-se o critério do Sebrae (2013), no qual microempresas são aquelas que possuem até dezenove funcionários, pequenas entre vinte a noventa e nove, médias entre cem a quatrocentos e noventa e nove, e grandes aquelas que possuem quinhentos funcionários ou mais.

Tabela 2 - Frequência de empresas por porte

\begin{tabular}{lcc}
\hline Porte & $\begin{array}{l}\text { Frequência } \\
\text { absoluta }\end{array}$ & Frequência relativa (\%) \\
\hline Micro & 10 & 12,7 \\
Pequena & 22 & 27,8 \\
Média & 22 & 27,8 \\
Grande & 25 & 31,7 \\
\hline
\end{tabular}

\section{RESULTADOS E DISCUSSÕES}

Considerando o referencial teórico apresentado e discutido, a Tabela 3 apresenta os valores de média, desvio padrão e mediana para cada estímulo avaliado na amostra pesquisada. 
Tabela 3 - Estímulos para a adoção do ecodesign

\begin{tabular}{lccc}
\hline \multicolumn{1}{c}{ Variável } & Média & $\begin{array}{c}\text { Desvio } \\
\text { Padrão }\end{array}$ & Mediana \\
\hline Benefício para o meio ambiente. & 5,09 & 1,81 & 5 \\
Redução de custos. & 6,06 & 1,27 & 6 \\
Melhoria da imagem da empresa. & 5,92 & 1,21 & 6 \\
Novas oportunidades de mercado. & 6,18 & 1,19 & 7 \\
Aumento da qualidade do produto. & 5,92 & 1,37 & 6 \\
Cumprimento de requisitos legais. & 6,38 & 1,11 & 7 \\
Oportunidades de inovação. & 5,90 & 1,30 & 6 \\
Sinergia com outros requisitos dos produtos da empresa. & 5,65 & 1,35 & 6 \\
\hline
\end{tabular}

Ao se analisar a Tabela 3, observa-se por um lado que o cumprimento dos requisitos legais e as novas oportunidades de negócios receberam a maior pontuação dentre as investigadas (essas duas variáveis apresentam mediana com o valor sete), ou seja, são os quesitos que mais incentivam as empresas a adotarem o ecodesign. Por outro lado, o estímulo "benefício ao meio ambiente" apresentou a menor mediana dentre as variáveis pesquisadas (mediana valor cinco). A figura 1 ilustra esses resultados por meio de gráfico radar.

Figura 1 - Estímulos para a adoção do ecodesign.

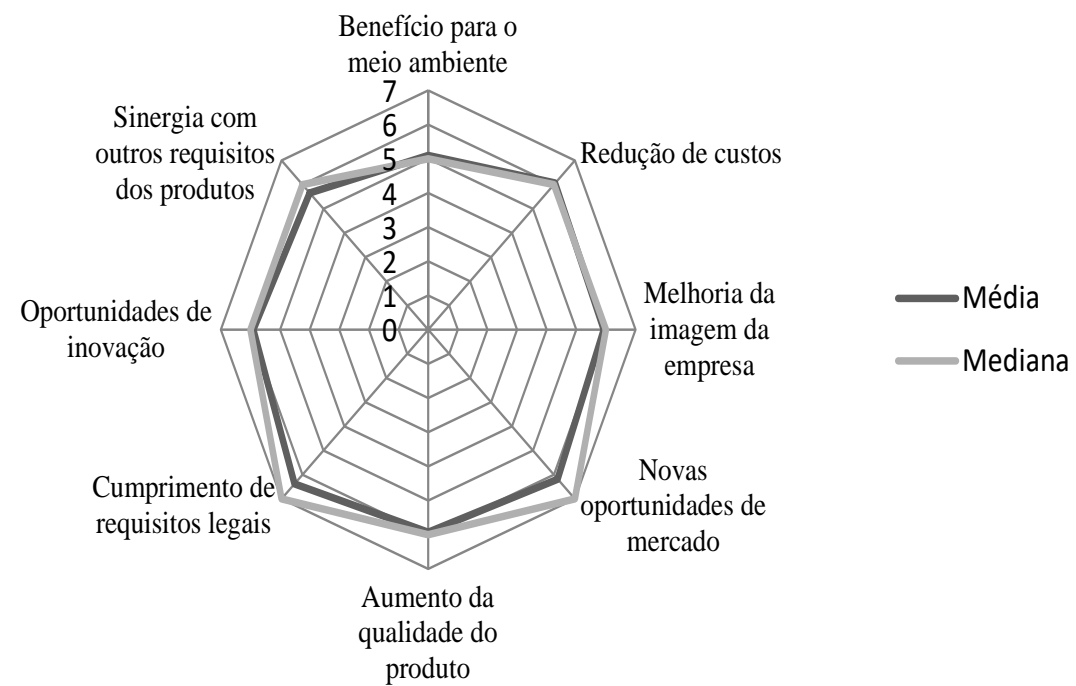

É possível visualizar tanto na tabela 1 quanto na Figura 1 que há concordância por parte das empresas com a importância dos estímulos investigados para a adoção do ecodesign, visto que as oito variáveis apresentam média e mediana entre cinco e sete. Este resultado indica que os estímulos sugeridos pela literatura e utilizado para compor o instrumento de pesquisa possuem efetiva influência sobre as decisões das empresas em empregar práticas ambientais no 
desenvolvimento de novos produtos.

Especificamente, verifica-se na figura 1 que o cumprimento dos requisitos legais e as novas oportunidades de negócios se sobressaem como estímulos para a adoção do ecodesign. Com relação ao atendimento aos requisitos legais, este resultado obtido é alinhado com a literatura em gestão ambiental, uma vez que o atendimento a aspectos legais é apontado tanto na literatura internacional (CLUZEL et al. 2016; DANGELICO, 2015; DALHAMMAR, 2016), quanto na nacional (JABBOUR; JABBOUR, 2016) como importante estímulo para a adoção do ecodesign. Por exemplo, conforme Jabbour e Jabbour (2013) mencionam que muitas empresas no Brasil somente reagem às leis ambientais impostas pelo governo. Nesse sentido, os acordos setoriais provenientes da Política Nacional de Resíduos Sólidos no Brasil não exigem o uso específico de métodos e ferramentas de ecodesign, mas estes métodos e ferramentas tendem a auxiliar na adequação dos produtos e processos a esses requisitos legais. E, conforme demonstram os resultados desta pesquisa, a legislação efetivamente possui impacto significativo no estímulo para a adoção de práticas ambientais associadas a produto.

Da mesma forma, o potencial do ecodesign e a preocupação ambiental no desenvolvimento de novos produtos, também é reconhecido como meio que proporciona novas oportunidades de mercado (DANGELICO, 2015; DANGELICO et al., 2013; PIGOSSO et al., 2013). Estudos pontuam (DANGELICO et al., 2013; DANGELICO et al., 2016) que o desenvolvimento de produtos ambientalmente sustentável possui potencial para ampliar a participação em mercados em que a empresa já atua; ou, também, para atender novos mercados, especialmente, àqueles que demandam uma postura mais proativa de empresas no que se refere à sustentabilidade ambiental

A posssibilidade de utlizar o ecodesign como elemento para a redução de custos de produção também foi estímulo considerado relevante pelas empresas pesquisadas (média de 6,02). Possivelmente, o menor dispêndio de energia, água, e reuso de materiais, por exemplo, esta relacionado com este resultado. De maneira alinhada com as propstas de Porter e Van der Linde (1995) esta observação indica também que essa preocupação ambiental no desenvolvimento de produtos gera benefícios financeiros operacionais e, portanto, são potenciais atrativos para as 
empresas adotarem o ecodesign. Dentre os estímulos internos, o ecodesign pode ser utilizado como meio para a redução de custos e a melhoria da qualidade dos produtos. Por outro lado, observa-se que o estímulo "benefício ao meio ambiente" apresentou a menor média dentre as variáveis pesquisadas.

Vale destacar também que na literatura existem resultados divergentes aos destacados aqui. Por exemplo, alguns estudos (KNIGHT; JENKINS, 2009; VAN HEMEL; CRAMER, 2002) destacam que estímulos como a necessidade de inovar, melhorar a qualidade dos produtos, explorar novos mercados e, até mesmo o atendimento a requisitos legais tendem a ser mais fracos do que estímulos internos como redução de custos, o benefício ambiental obtido e a melhoria de imagem da empresa. Entende-se que esta divergência de resultados entre as empresas que atuam no Brasil em relação ao já observado por pesquisas internacionais poderiam ser exploradas de maneira mais aprofundada em estudos futuros.

A tabela 4 compara os resultados gerais (média, mediana e desvio padrão para todos os estímulos conjuntamente) considerando o porte das empresas.

Tabela 4 - Estímulos para a adoção do ecodesign considerando o porte da empresa

\begin{tabular}{lccc}
\hline \multicolumn{1}{c}{ Porte } & Média & Mediana & Desvio Padrão \\
\hline Micro & 6,1 & 6,5 & 0,95 \\
Pequeno & 6 & 6,5 & 0,92 \\
Médio & 5,6 & 6 & 1,15 \\
Grande & 6 & 6,5 & 0,99 \\
\hline
\end{tabular}

Apesar dos resultados serem próximos, observa-se na tabela 2 que parte das empresas de médio porte são as menos sensíveis aos estímulos pesquisados. Detalham-se esses resultados na Tabela 5 , que compara as oito variáveis com o porte das empresas.

Ao se analisar as tabelas 4 e 5, nota-se que as microempresas são as mais sensíveis a adoção do ecodesign. O que ocorre, principalmente, devido aos estímulos legais e pelas possibilidades de novas oportunidades de mercado e de inovação. Sabendo-se que a demanda de mercados consumidores pelos produtos sustentáveis ambientalmente tem crescido (DANGELICO et al., 2016; DANGELICO; et al., 2013; VAN HEMEL; CRAMER, 2002),esse é um estímulo que parece também estar influenciando as empresas que participaram desta pesquisa, sobretudo as 
menores, em adotar o ecodesign. Ademais, estudos anteriores sugerem que estruturas organizacionais dinâmicas, normalmente, presentes nas micro e pequenas empresas possuem a vantagem de maior agilidade (BOCKEN et al., 2014), convergindo também aos resultados aqui apresentados. Sendo assim, a eco inovação aplicada aos produtos também pode ser mais adequada para as empresas pequenas, iniciantes ou até mesmo as de base tecnológica.

Tabela 5 - Comparação entre as variáveis pesquisadas e porte da empresa.

\begin{tabular}{|c|c|c|c|c|c|c|c|c|}
\hline \multirow[t]{3}{*}{ Variável } & \multicolumn{8}{|c|}{ Tamanho da Empresa } \\
\hline & \multicolumn{2}{|c|}{ Micro } & \multicolumn{2}{|c|}{ Pequeno } & \multicolumn{2}{|c|}{ Médio } & \multicolumn{2}{|c|}{ Grande } \\
\hline & Média & Mediana & Média & Mediana & Média & Mediana & Média & Mediana \\
\hline $\begin{array}{l}\text { Benefício para o meio } \\
\text { ambiente }\end{array}$ & 5,5 & 5,5 & 5,1 & 5,5 & 4,8 & 5 & 5,2 & 5 \\
\hline Redução de custos & 6,1 & 6,5 & 6 & 6,5 & 5,9 & 6 & 6,2 & 6 \\
\hline $\begin{array}{l}\text { Melhoria da imagem da } \\
\text { empresa }\end{array}$ & 5,8 & 6 & 6,1 & 6 & 5,7 & 6 & 6 & 6 \\
\hline $\begin{array}{l}\text { Novas oportunidades } \\
\text { de mercado }\end{array}$ & 6,4 & 7 & 6,4 & 7 & 5,8 & 6 & 6,3 & 7 \\
\hline $\begin{array}{l}\text { Aumento da qualidade } \\
\text { do produto }\end{array}$ & 6,1 & 6,5 & 6,1 & 7 & 5,2 & 6 & 6,1 & 6 \\
\hline $\begin{array}{l}\text { Cumprimento de } \\
\text { requisitos legais }\end{array}$ & 6,4 & 7 & 6,4 & 7 & 6,3 & 7 & 6,5 & 7 \\
\hline $\begin{array}{l}\text { Oportunidades de } \\
\text { inovação }\end{array}$ & 6,3 & 7 & 6,01 & 6 & 5,4 & 6 & 6 & 7 \\
\hline $\begin{array}{l}\text { Sinergia com outros } \\
\text { requisitos dos produtos } \\
\text { da empresa }\end{array}$ & 6 & 7 & 5,9 & 6 & 5,3 & 5 & 5,7 & 6 \\
\hline
\end{tabular}

Verifica-se também que estímulo "benefício para o meio ambiente" teve o menor resultado, especialmente, entre as empresas de médio porte (valores de média e de mediana). Vale destacar que o estímulo relacionado à sinergia com os outros produtos, teve pontuação abaixo das demais nas empresas de grande porte. Como são justamente estas empresas que normalmente possuem portfólio mais amplo de produtos, e que compartilham características a partir de produtos plataformas e derivativos, esperava-se que esta variável tivesse uma pontuação maior especialmente entre as firmas de grande porte, o que não foi observado.

\section{CONCLUSÕES}

Ao identificar e analisar práticas e estímulos à adoção do ecodesign em uma amostra de empresas pertencentes a setores inovadores no país, e que estão envolvidas em atividades de desenvolvimento de novos produtos, este artigo 
contribui em áreas como gestão ambiental, gestão de desenvolvimento de produtos e gestão de projetos. Pela frequência observada nas respostas das 79 empresas pesquisadas, pode-se observar inicialmente que de maneira geral, há concordância de que estímulos citados pela literatura, sobretudo internacional, representam elementos motivadores para a adoção das estratégias de ecodesign no desenvolvimento de novos produtos, uma vez que para cada estímulo citado foi constatada uma concordância maior ou igual a 70\% (responderam 5, 6 ou 7 para os estímulos) e média e mediana maiores que 5.

Observou-se que a adequação à requisitos legais é um estímulo importante para a adoção do ecodesign nas empresas dos diferentes portes e que atuam no Brasil. A disseminação deste resultado pode ser uma importante informação para os formuladores de políticas públicas, uma vez que os resultados desta pesquisa sugerem que a legislação efetivamente estimula grande parte dos esforços das empresas para a adoção do ecodesign, o que por sua vez gera externalidades ambientais positivas. Por outro lado, o "benefício para o meio ambiente" foi a variável com menor mediana entre as investigadas. Mesmo reconhecendo que este é um resultado que merece ser investigado de maneira mais detalhada em pesquisas futuras, a menor importância conferida a esta variável somada aos demais resultados aqui apresentados e discutidos sugerem que as empresas no país são mais direcionadas pelos estímulos externos para a adoção do ecodesign (oportunidade de mercado e adequação a legislação, por exemplo), do que propriamente pelos estímulos internos.

De maneira geral, independentemente do porte, as empresas são seníveis aos estímulos para a adoção do ecodesign, o que fica claro quando se analisa os resultados presentes na tabela 2. Vale o destaque para os resultados apresentados pela microempresas, que demonstraram serem um pouco mais sensíveis em relação aos estímulos investigados. Talvez por serem empresas mais novas, possuam maior disposição para se posicionarem no mercado pela perspectiva ambiental, e com a intenção em diferenciar os seus produtos por meio do ecodesign. Pesquisas futuras, sob a forma de estudos de casos, poderiam se dedicar a explorar especificamente este fenômeno nas microempresas.

Por fim, reconhece-se que esse trabalho possui diversas limitações, tais como 
o perfil dos respondentes e o tamanho da amostra obtida. O uso de questões com ênfase em aspectos perceptivos e a seleção dos principais estímulos ao ecodesign para compor o instrumento de pesquisa também são elementos que limitam os resultados deste artigo. Entende-se, assim, que estudos futuros poderiam ampliar os resultados desta pesquisa e o corpo de conhecimento em ecodesign ao investigar outros setores, incluir novos fatores e variáveis; assim como correlacioná-los por meio de análise fatorial com aspectos de desempenho, tais como o operacional, inovador e de mercado.

\section{AGRADECIMENTOS}

Os autores gostariam de agradecer o apoio recebido pela Fundação de Amparo à Pesquisa do Estado de São Paulo, FAPESP (processo nº 15/00110-6).

\section{REFERÊNCIAS}

AMMENBERG, J.; SUNDIN, E. Products in environmental management systems: Drivers, barriers and experiences. Journal of Cleaner Production, v. 13, n. 4, p. $405-415,2005$. http://doi.org/10.1016/j.jclepro.2003.12.005

BABBIE, E. Métodos de pesquisas de Survey. Belo Horizonte: UFMG, 1999. 519p. BAUMANN, H.; BOONS, F.; BRAGD, A. Mapping the green product development field: Engineering, policy and business perspectives. Journal of Cleaner Production, v. 10, n. 5, p. 409-425, 2002. http://doi.org/10.1016/S0959-6526(02)00015-X

BOCKEN, N. M. P.; FARRACHO, M.; BOSWORTH, R.; KEMP, R. The front-end of ecoinnovation for eco-innovative small and medium sized companies. Journal of Engineering and Technology Management, v. 31, p. $43-57,2014$.

http://doi.org/10.1016/j.jengtecman.2013.10.004

BORCHARDT, M.; WENDT, M.H.; SELLITO, M.A.; PEREIRA,G.M. Reprojeto do contraforte: um caso de aplicação do ecodesign em uma empresa do setor calçadista. Production, $v$. 20, n. 3, p. 392-403, 2010. http://dx.doi.org/10.1590/S0103-65132010005000006

BRONES, F.; CARVALHO, M. M. From 50 to 1: integrating literature toward a systemic ecodesign model. Journal of Cleaner Production, v. 96, 1, p. 44-57, 2015.

http://doi.org/10.1016/j.jclepro.2014.07.036

BRONES, F.; DE CARVALHO, M. M.; DE SENZI ZANCUL, E. Ecodesign in project management: A missing link for the integration of sustainability in product development? Journal of Cleaner Production, v. 80, October, p. 106-118, 2014. http://doi.org/10.1016/j.jclepro.2014.05.088 
BOVEA, M. D.; PÉREZ-BELIS, V. A taxonomy of ecodesign tools for integrating environmental requirements into the product design process. Journal of Cleaner Production, v. 20, n.1 p. 61-71, 2012. http://doi.org/10.1016/j.jclepro.2011.07.012

BYGGETH, S.; HOCHSCHORNER, E. Handling trade-offs in Ecodesign tools for sustainable product development and procurement. Journal of Cleaner Production, v. 14, n. 15-16, p. 1420-1430, 2006. http://doi.org/10.1016/j.jclepro.2005.03.024

CAUCHICK MIGUEL, P.A; HO, L. H. Levantamento tipo survey. Metodologia de pesquisa em engenharia de produção e gestão de operações. 1. ed. Rio de Janeiro: Elsevier, 2010. p. 73-128.

CLUZEL, F.; YANNOU, B.; MILLET, D.; LEROY, Y. Eco-ideation and eco-selection of R\&D projects portfolio in complex systems industries. Journal of Cleaner Production, v. 112, n. 5, p. 4329 - 4343, 2016. http://doi.org/10.1016/j.jclepro.2015.08.002

COLLADO-RUIZ, D.; OSTAD-AHMAD-GHORABI, H. Estimating environmental behavior without performing a life cycle assessment. Journal of Industrial Ecology, v. 17, n.1, p. 31-42, 2013. http://doi.org/10.1111/j.1530-9290.2012.00510.x

DALHAMMAR, C. Industry attitudes towards ecodesign standards for improved resource efficiency. Journal of Cleaner Production, v.123, n.1, p.155-166, 2016.

http://doi.org/10.1016/j.jclepro.2015.12.035

DANGELICO, R. M. Green product innovation: where we are and where we are going. Business Strategy and the Environment, v.8, n. 25, p. $560-576,2015$. http://doi.org/10.1002/bse.1886

DANGELICO, R. M.; PONTRANDOLFO, P.; PUJARI, D. Developing sustainable new products in the textile and upholstered furniture industries: role of external integrative capabilities. Journal of Product Innovation Management, v. 30, n. 4, p. 642-658, 2013. http://doi.org/10.1111/jpim.12013

DANGELICO, R. M.; PONTRANDOLFO, P.; PUJARI, D. Green product innovation in manufacturing firms: a sustainability-oriented dynamic capability perspective. Business Strategy and Environment, in press, 2016. http://doi.org/10.1002/bse.1932

DANGELICO, R. M.; PUJARI, D. Mainstreaming green product innovation: Why and how companies integrate environmental sustainability. Journal of Business Ethics, v. 95, n. 3, p. 471-486, 2010. http://doi.org/10.1007/s10551-010-0434-0

DEKONINCK, E. A.; DOMINGO, L.; O'HARE, J. A.; PIGOSSO, D.A.; REYES, T.; TROUSSIER, N. Defining the challenges for ecodesign implementation in companies: development and consolidation of a framework. Journal of Cleaner Production, v. 135,n. 1, p. 410-425, 2016. http://doi.org/10.1016/j.jclepro.2016.06.045

EPPINGER, S. The fundamental challenge of product design. Journal of Product Innovation Management, v. 28, p. 399-400, 2011. http://doi.org/10.1111/j.1540$\underline{5885.2011 .00810 . x}$

FIKSEL, J. Design for Environment. New York: Mc Graw Hill, 1996. 432p. 
FORZA, C. Survey research in operations management: a process-based perspective.

International Journal of Operations \& Production Management v. 22, n. 2, p. 152-194, 2002. http://dx.doi.org/10.1108/01443570210414310

GONZÁLEZ-BENITO, J.; GONZÁLEZ-BENITO, Ó. A review of determinant factors of environmental proactivity. Business Strategy and the Environment, v. 15, n. 2, p. 87-102, 2006. http://doi.org/10.1002/bse.450

GRAEDEL, T.E.; ALLENBY, B.R. Industrial ecology. Prentice Hall, 1995, 363p.

IBGE. PINTEC - Pesquisa de Inovação Tecnológica. Rio de Janeiro: 2013

JABBOUR, A. B. L. S; JABBOUR, C. J. C. Gestão ambiental nas organizações: fundamentos e tendências. São Paulo: Atlas, 2013. 104p.

JABBOUR, C. J. C.; JUGEND, D.; JABBUR, A.B.L.S.; GUNASEKARAN, A.; LATHAM, H. Green product development and performance of Brazilian firms: measuring the role of human and technical aspects. Journal of Cleaner Production, v. 87, p. 442-451, 2015. http://doi.org/10.1016/.j.jclepro.2014.09.036

JOHANSSON, G. Success factor for integration of Ecodesign in product development: a review of state of the art. Environment Management and Health, Wagon Lane, v. 13, n. 1, p 98-107, 2002. http://dx.doi.org/10.1108/09566160210417868

KNIGHT, P.; JENKINS, J. O. Adopting and applying eco-design techniques: a practitioners perspective. Journal of Cleaner Production, v. 17, n. 5, p. 549-558, 2009.

http://doi.org/10.1016/j.jclepro.2008.10.002

KUBOTA, F.S.; CAMPOS, L. M.S.; CAUCHICK MIGUEL, P.A. Uma análise preliminar das contribuições da modularidade em produto ao ecodesign. Produção on line, v.14, n. 2, p. 560-592, 2014. http://dx.doi.org/10.14488/1676-1901.v14i2.1424

LU, B.; ZHANG, J.; XUE, D.; GU, P. Systematic lifecycle design for sustainable product development. Concurrent Engineering: Research and Applications, v19, n.4, 307-324, 2011. http://doi.org/10.1177/1063293X11424513

LUIZ, O. R.; JUGEND, D.; JABBOUR, C.J.C.; LUIZ, O., SOUZA. F. B. Ecodesign field of research throughout the world: mapping the territory by using an evolutionary lens.

Scientometrics, v. 109, n.1, p. 241- 59, 2016. http://doi.org/10.1007/s11192-016-2043-x

LUTTROPP, C.; LAGERSTEDT, J. EcoDesign and The Ten Golden Rules: generic advice for merging environmental aspects into product development. Journal of Cleaner Production, v. 14, n. 15/16, p. 1396-1408, 2006.

http://doi.org/10.1016/j.jclepro.2005.11.022

MAGNAGO, P.F.; AGUIAR, J.P.O; PAULA, I.C. Sustentabilidade em desenvolvimento de produtos: uma proposta para a classificação de abordagens. Revista Produção online, v. 12, n.2, 351-376, 2012. http://dx.doi.org/10.14488/1676-1901.v12i2.796

PIGOSSO, D. C. A.; ROZENFELD, H.; MCALOONE, T. C. Ecodesign maturity model: a management framework to support ecodesign implementation into manufacturing companies. Journal of Cleaner Production, v. 59,n.15, 160-173, 2013.

http://doi.org/10.1016/j.jclepro.2013.06.040 
PORTER, M.E.; VAN DER LINDE, C. Green and competitive: ending the stalemate. Harvard Business Review, v. 28, n. 6, p. 160-173, 1995.

POULIKIDOU, S.; BJÖRKLUND, A.; TYSKENG, S. Empirical study on integration of environmental aspects into product development: Processes, requirements and the use of tools in vehicle manufacturing companies in Sweden. Journal of Cleaner Production, v. 81, n. 15, p. 34-45, 2014. http://doi.org/10.1016/j.jclepro.2014.06.001

PUJARI, D. Eco-innovation and new product development: Understanding the influences on market performance. Technovation, v. 26, n. 1, p. 76-85, 2006.

http://doi.org/10.1016/j.technovation.2004.07.006

ROZENFELD, H.; FORCELLINI, F. A.; AMARAL, D. C.; TOLEDO, J. C.; SILVA, S. L.; ALLIPRANDINI, D. H.; SCALICE, R.; K. Gestão de desenvolvimento de produto: uma referência para a melhoria do processo. São Paulo: Saraiva, 2006.

SEBRAE. Critérios de classificação de empresas: EI - ME - EPP. Disponível em: http://www.sebrae-sc.com.br/leis/default.asp?vcdtexto=4154. Acessado em e julho de 2013.

SELLITO, M.A.; BORCHARDT, M; PEREIRA, G.M.; PACHECO, D.A.J. Gestão de cadeias de suprimentos verdes: quadro de trabalho. Revista Produção Online, v.13, n. 1, p. 351374, 2013. http://dx.doi.org/10.14488/1676-1901.v13i1.1181

STANDARD, I. ISO 14006:2011 - Environmental management systems - Guidelines for incorporating ecodesign, 2011.

STEVELS, A. Integration of ecodesign into business, a new challenge. In: ENVIRONMENTALLY CONSCIOUS DESIGN AND INVERSE MANUFACTURING SYMPOSIM, 1., 1999, Los Alamitos. Anais.. Los Alamitos: IEE Computer Society, 1999. p. 27-35.

VAN HEMEL, C.; CRAMER, J. Barriers and stimuli for ecodesign in SMEs. Journal of Cleaner Production, v. 10, n. 5, p. 439-453, 2002. http://doi.org/10.1016/S0959$\underline{6526(02) 00013-6}$

VARANDAS JUNIOR, A. Uma proposta para integração de aspectos ambientais do ecodesign no processo de desenvolvimento de novos produtos. Tese de doutorado. Escola Politécnica da Universidade de São Paulo, 2014.

VERCALSTEREN, A. Integrating the ecodesign concept in small and medium-size enterprises: experiences in the Flemish region of Belgium. Environmental Management and Health, v. 12, n. 4, p. 347-355, 2001. http://dx.doi.org/10.1108/EUM0000000005706

WALKER, H.; SISTOB, L.; MCBAINC, D. Drivers and barriers to environmental supply chain management practices: lessons from the public and private sectors. Journal of Purchasing \& Supply Management, v.14, n.1, p.69-85, 2008.

http://doi.org/10.1016/i.pursup.2008.01.007

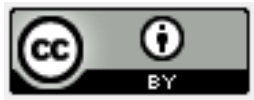

Artigo recebido em 29/12/2016 e aceito para publicação em 18/04/2017

DOI: http://dx.doi.org/10.14488/1676-1901.v17i2.2676 